Résumés des conférences et travaux

\title{
Histoire des doctrines stratégiques
}

Hervé Coutau-Bégarie

\section{OpenEdition \\ Journals}

Édition électronique

URL : https://journals.openedition.org/ashp/483

DOI : 10.4000/ashp.483

ISSN : 1969-6310

Éditeur

Publications de l'École Pratique des Hautes Études

\section{Édition imprimée}

Date de publication : 1 octobre 2008

Pagination : 288-289

ISSN : 0766-0677

Référence électronique

Hervé Coutau-Bégarie, "Histoire des doctrines stratégiques ", Annuaire de l'École pratique des hautes études (EPHE), Section des sciences historiques et philologiques [En ligne], 139 | 2008, mis en ligne le 26 novembre 2008, consulté le 12 juillet 2021. URL : http://journals.openedition.org/ashp/483 ; DOI https://doi.org/10.4000/ashp.483 


\title{
HISTOIRE DES DOCTRINES STRATÉGIQUES
}

\author{
Directeur d'études : M. Hervé Coutau-BÉGarie
}

Programme de l'anéee 2006-2007 : I. La diplomatie de la force. — II. Histoire militaire des guerres de Vendée.

Depuis quelques années, la conférence est malheureusement troublée par des contingences extérieures qui empêchent le déroulement du programme prévu. Après les manifestations d'agitateurs contre le CPE l'an dernier, c'est une hospitalisation du directeur d'études qui a entraîné la suspension de la conférence pendant quatre mois. En conséquence, il n'a pas été possible de traiter les deux sujets annoncés qui ont été renvoyés à l'année prochaine.

Les conférences du premier trimestre ont été consacrées, comme l'habitude en a été prise depuis plusieurs années, à un survol de l'actualité stratégique. Outre l'intérêt très vif des auditeurs pour cet exercice, cela permet de rapporter l'actualité aux grands débats théoriques et d'essayer de mesurer la pertinence des théories classiques à l'aune des transformations accélérées auxquelles nous sommes aujourd'hui confrontés.

Les guerres d'Irak et d'Afghanistan ne peuvent qu'inciter à une réflexion sur les conflits asymétriques et donc sur l'intérêt de classiques presque tous conçus dans la perspective de la grande guerre, appelée ensuite stratégie conventionnelle et aujourd'hui conflits symétriques. Les affaires iranienne et nord-coréenne, d'une part, le développement des anti-missiles, d'autre part, posent de la même manière le problème de la mutation de la stratégie nucléaire qui ne se limite plus à la dissuasion mais recouvre désormais une palette beaucoup plus large allant de la prévention à la protection sans exclure l'éventualité de la coercition avec le recours aux mini-nukes.

Après la reprise de la conférence en avril, l'actualité a de nouveau été abordée, sous l'angle spécifique de la politique française de la défense, en raison des inévitables ajustements ou ruptures qu'elle subira du fait de l'élection présidentielle. Un inventaire des moyens a été esquissé, faisant ressortir la quasi impossibilité de se conformer au modèle d'armée 2015, à moins d'un effort financier considérable qui semble aujourd'hui hors de propos. Une adaptation est-elle concevable qui permettrait de parvenir à un effort financier acceptable sans sacrifier aucune des capacités majeures? Cette question, très pratique, incite à revenir sur l'éternel problème du primat de la technique et de la tyrannie des moyens par rapport aux fins. Cet examen ne fait pas ressortir des conclusions très encourageantes dans la mesure où quelles que soient les stratégies déclaratoires, l'amoindrissement des moyens a nécessairement des conséquences opérationnelles.

Enfin, quelques séances ont été consacrées à la poursuite de l'étude des théories des relations internationales pour les rapprocher de la théorie stratégique. L'impression dominante est négative : les raffinements méthodologiques croissants ne débouchent 
pas sur une plus-value opératoire correspondante. En termes plus brutaux, la prolifération d'un discours souvent jargonnant ne favorise pas une meilleure intelligence de la réalité. Le détour par la théorie des relations internationales ne fait que confirmer la pertinence du retour aux classiques. 